\title{
An Integer Programming Model and a Tabu Search Algorithm to Generate $\alpha$-labeling of Special Classes of Quadratic Graphs
}

\author{
M. Salarrezaei ${ }^{1}$, K. Eshghi ${ }^{2, *}$ \\ First, an integer programming model is proposed to find an $\alpha$-labeling for quadratic graphs \\ $Q(m, 4 k)$. Then, a Tabu search algorithm is developed to solve large scale problems. The \\ proposed approach can generate $\alpha$-labeling for special classes of quadratic graphs \\ $Q(m, 4 k), 6 \leq m \leq 10,2 \leq k \leq 10$, not previously reported in the literature. Then, the main \\ theorem of the paper is presented. We show how a problem in graph theory can be modeled and \\ solved by an integer programming model and a metaheuristic approach.
}

Keywords: Graph labeling, $\alpha$-labeling, Quadratic graphs, Integer programming, Tabu search algorithm.

Manuscript was received on 01/01/2017 revised on 05/03/2017 and accepted for publication on 13/05/2017

\section{Introduction}

Suppose that $G=(V, E)$ is a simple graph without loop and multiple edges which has $|V|$ vertices and $n=|E|$ edges. A graceful labeling for $G$ is a one-to-one mapping $\Psi$ from the vertex set $V(G)$ to the set $\{0,1,2, \ldots, n\}$ such that for each edge $e \in E(G)$ which connects the vertices $u, v \in V(G)$ to each other, the value $\bar{\Psi}(e)=|\Psi(u)-\Psi(v)|$ is a one-to-one mapping from the set of edges $E(G)$ to the set $\{1,2, \ldots, n\}$. A graph that has a graceful labeling is called a graceful graph [17].

Furthermore, graph $G$ has an $\alpha$-labeling if and only if

1- it is graceful, and

2- there is a number $\gamma, 0 \leq \gamma \leq|E(G)|$, such that for each edge $e \epsilon E(G)$ with two incident vertices $u, v \in V(G)$, the following condition holds:

$$
\min [\Psi(u), \Psi(v)] \leq \gamma<\max [\Psi(u), \Psi(v)] .
$$

\footnotetext{
* Corresponding Author.

1 Department of Industrial Engineering, Sharif University of Technology, Tehran, Iran, Email: m_salarrezaei@alum.sharif.edu.

2 Department of Industrial Engineering, Sharif University of Technology, Tehran, Iran, Email: eshghi@sharif.edu.
} 


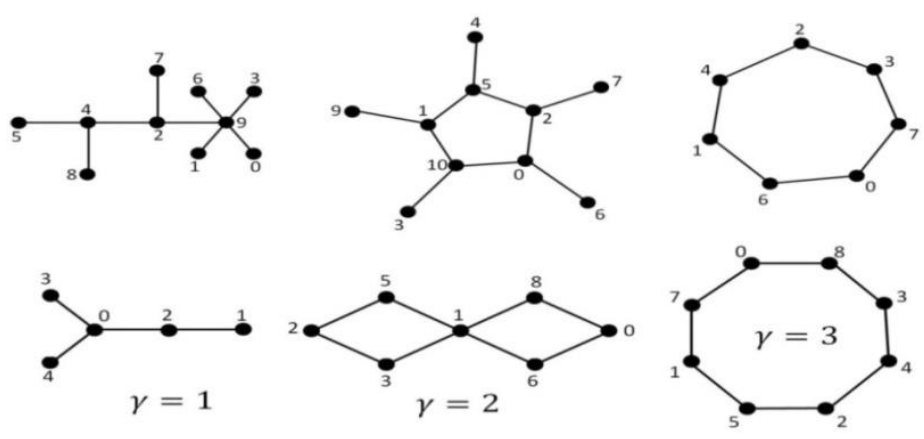

Figure 1. Six graceful graphs, three of them having $\boldsymbol{\alpha}$-labeling

The number $\gamma$ is named as "middle value". Suppose that a graph has $\alpha$-labeling, if a vertex label is smaller than $\gamma$ then it is referred as a "small vertex" and if it is greater than $\gamma$ then it is called a "large vertex". In Fig. 1, six graceful graphs are shown including three graphs with $\alpha$-labeling [15]. A cycle with 8 vertices in this figure has $\alpha$-labeling with small vertices $0,1,2$ and large vertices 4,5,7,8.

Graceful labeling and $\alpha$-labeling were first introduced by Rosa [17] and since then many graphs have been proved to have graceful or $\alpha$-labeling. The determination of which graphs are graceful or having $\alpha$-labeling has attracted a lot of interest. The conjecture that all trees are graceful is the bestknown open problem in the area of graph labeling. Although many papers have been published on the subject of graph labeling, there are a few particular techniques to use by authors. The graph labeling problem is to find out whether a given graph has a certain type of labeling and if it has it how to label the vertices. The common approach for proof is to construct desired labeling for all cases either by providing a formula or by generating all possible cases. Unfortunately, the process of proving a certain type of labeling by a particular graph $G$ is a very tedious and difficult task for many classes of graphs. The comprehensive studies on various types of labeling and existence of them for different types of graphs can be found in $[9,10]$. Here, finding $\alpha$-labeling for a special class of graphs, called quadratic graphs, is discussed. A quadratic graph $Q(m, 4 k)$ is a 2-regular graph with $m$ components, each of which is a cycle of length $4 k$. For example, an $\alpha$-labeling of $Q(2,4)$ is shown in Fig. 2 with $\gamma=4$.

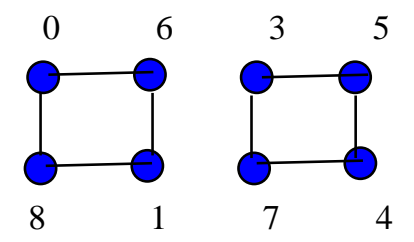

Figure 2. an $\boldsymbol{\alpha}$-labeling of $\boldsymbol{Q}(2,4)$

Some of the results found for the quadratic graphs so far are as follows:

- $\quad$ Graph $Q(1, n)$ is graceful if and only if $n \equiv 0$ or $3 \bmod (4)$. Furthermore, if it has an $\alpha$ labeling, then $n \equiv 0 \bmod (4)$ [17].

- $\quad$ For $k>0$ and $k \neq 3$, graph $Q(k, 1)$ has an $\alpha$-labeling. Graph $Q(3,1)$ is graceful but does not have an $\alpha$-labeling [1].

- $\quad$ For $k \geq 2, Q(3,4 k)$ has an $\alpha$-labeling [13].

- $\quad$ For $k \geq 1, Q(4,4 k)$ has an $\alpha$-labeling [14]. 
- $\quad$ For $k \geq 1, Q(5,4 k)$ has an $\alpha$-labeling [5].

- $\quad$ All bipartite graphs, except $Q(3,1)$, that satisfies Rosa's condition $(m \equiv 0 \bmod (4))$ and has at most 24 vertices, has an $\alpha$-labeling [2].

In previous studies, most results are either theoretical proofs or ad hoc methods to obtain labeling of graphs. Among many papers in this area, only a few of them focus on developing mathematical models or algorithms to obtain the labeling; see Redl [16], Eshghi and Azimi [7,8], Smith [18], Smith and Puget [19], Mahmoudzadeh and Eshghi [15]. The authors used different mathematical programming models or approaches to formulate and solve the problem such as integer programming $[7,8]$, nonlinear programming [16], constraint programming [18,19] and ant colony optimization algorithm [15]. In all of these studies, the authors construct a model to find labeling for special classes of graphs such as trees, cycles, crowns, etc. Furthermore, their models have some limitations on the size of graphs and can be applied only on graphs having at most forty vertices.

The rest of our work is organized as follows: in Section 2, an integer programming model is developed for solving the problem and in Section 3, a proposed tabu search is described. In Section 4 , some details of a labeling procedure are described. Computational results and conclusion are respectively presented in the Sections 5 and 6. In the appendix, the details of $\alpha$-labeling of $Q(m, 4 k)$ for $6 \leq m \leq 10$ and $2 \leq k \leq 10$, are presented.

\section{Mathematical Formulation of the Problem}

In order to formulate the problem, first a corollary obtained by Abraham and Kotzig [3] is used as follows: if graph $G$ is 2-regular with $n$ vertices/edges, $n=4 k$, and $\Psi$ is an $\alpha$-labeling on $G$, then there is exactly one value $x(0<x<n)$ such that for every $v \in V(G), \Psi(v) \neq x$. This value is known as the missing value of the graceful labeling $\Psi$.

Using this result, the middle value $\gamma$ can be obtained as follows:

- If the missing value $x$ is equal to $k$, then $2 k \leq \gamma<2 k+1$ and $\gamma=2 k$.

- If the missing value is equal to $3 k$, then $2 k-1 \leq \gamma<2 k$ and $\gamma=2 k-1$.

Graph $Q(m, 4 k)$ has $4 m k$ vertices and $4 m k$ edges and in order to formulate the problem, it is assumed that $x=3 m k$ and $\gamma=2 m k-1$. Therefore, the vertices should be alternatively bigger or smaller than $\gamma$. Thus, in order to label the vertices, two groups of variables are defined as follows:

- $x_{i}, i=1,2, \ldots, 2 m k$, for small vertices' labels.

- $y_{i}, i=1,2, \ldots, 2 m k$, for large vertices' labels.

Moreover, $4 m k$ variables denoted by $e_{i}, i=1,2, \ldots, 4 m k$, are defined to label each an edge.

The labeling and ordering of the vertices and edges in $Q(m, 4 k)$ are respectively as follows:

$$
\begin{array}{lll}
\text { cycle } i: & x_{2(i-1) k+1}, y_{2(i-1) k+1}, x_{2(i-1) k+2}, y_{2(i-1) k+2}, \ldots, x_{2 i k}, y_{2 i k}, & i=1,2, \ldots, m, \\
\text { cycle } i: & e_{4(i-1) k+1}, e_{4(i-1) k+2}, \ldots, e_{4 i k}, & i=1,2, \ldots, m .
\end{array}
$$

Variable $x_{i}$ must be a discrete value from the set $X v a l=\{0,1,2, \ldots, 2 m k-1\}$ and as a result, variable $y_{i}$ must assume a value from the set Yval $=\{2 m k, 2 m k+1, \ldots, 3 m k-1,3 m k+$ 
$1,3 m k+2, \ldots, 4 m k\}$. Finally, the variable $e_{i}$ must take a value from the set Eval $=\{1,2, \ldots, 4 m k\}$. Moreover, the binary variables $w_{i j}$ and $r_{i j}$ are defined as follows:

$$
\begin{aligned}
& w_{i j}=\left\{\begin{array}{ccc}
1, & \text { if } x_{i}=X \operatorname{val}[j], & i=1,2, \ldots, 2 m k \\
0, & \text { o. } w ., & j=1,2, \ldots, 2 m k
\end{array}\right. \\
& w_{i, j+2 m k}=\left\{\begin{array}{ccc}
1, & \text { if } y_{i}=\operatorname{Yval}[j], & i=1,2, \ldots, 2 m k \\
0, & \text { o. } w_{.}, & j=1,2, \ldots, 2 m k
\end{array}\right. \\
& r_{i j}=\left\{\begin{array}{ccc}
1, & \text { if } e_{i}=\operatorname{Eval}[j], & i=1,2, \ldots, 4 m k \\
0, & \text { o.w. } & j=1,2, \ldots, 4 m k .
\end{array}\right.
\end{aligned}
$$

Using the above variables, the constraints of the problem are formulated as follows:

$$
\begin{array}{ll}
e_{2 i-1}=y_{i}-x_{i}, & i=1,2, \ldots, 2 m k \\
e_{2 i}=y_{i}-x_{i+1}, & i=1,2, \ldots, 2 m k, i \neq 2 k, 4 k, \ldots, 2 m k \\
e_{2 i}=y_{i}-x_{i+1-2 k}, & i=2 k, 4 k, \ldots, 2 m k \\
x_{i}=\sum_{j=1}^{2 m k} \text { Xval }[j] * w_{i j}, & i=1,2, \ldots, 2 m k \\
\sum_{j=1}^{2 m k} w_{i j}=1, & i=1,2, \ldots, 2 m k \\
\sum_{i=1}^{2 m k} w_{i j}=1, & j=1,2, \ldots, 2 m k \\
y_{i}=\sum_{j=1}^{2 m k} Y v a l[j] * w_{i}(j+2 m k), & i=1,2, \ldots, 2 m k \\
\sum_{j=2 m k+1}^{4 m k} w_{i j}=1, & i=1,2, \ldots, 2 m k \\
\sum_{i=1}^{2 m k} w_{i j}=1, & j=2 m k+1,2 m k+2, \ldots, 4 m k \\
e_{i}=\sum_{j=1}^{4 m k} E v a l[j] * r_{i j}, & j=1,2, \ldots, 4 m k \\
\sum_{j=1}^{4 m k} r_{i j}=1, & i=1,2, \ldots, 4 m k \\
\sum_{i=1}^{4 m k} r_{i j}=1, & j=1,2, \ldots, 4 m k \\
x_{i}, y_{i} \geq 0, \text { integer, } & i=1,2, \ldots, 2 m k \\
e_{i} \geq 0, \text { integer, } & i=1,2, \ldots, 4 m k \\
w_{i j} \in\{0,1\} & i=1,2, \ldots, 2 m k, j=1,2, \ldots, 4 m k \\
r_{i j} \in\{0,1\} &
\end{array}
$$

In this formulation, the constraints (6)-(8) identify the relationships between the vertex and edge labels according to equations (1) and (2). Constraint (9) specifies the admissible domain for the $x_{i}$ variables. Constraint (10) assigns a label for each vertex variable $x_{i}$. Constraint (11) ensures that each label is assigned to only one vertex. Equations (12)-(17) use the same trend to assign labels to the variables $y_{i}$ and $e_{i}$ respectively. Constraint (18) defines the type of variables used in the formulation. 
Variables $x, y$ and $e$ are defined as integer variables; however, considering equations (9), (12), (15), this integer condition can be dropped.

Lemma 1. In the proposed model, the constraint (16) always holds and can be removed from the formulation.

Proof by contradiction. Assume that by dropping the constraint (16), this constraint is violated, meaning that there is $i \in\{1,2, \ldots, 4 m k\}$ such that $\sum_{j=1}^{4 m k} r_{i j} \neq 1$.

Constraints (9)-(14) force the variables $x_{i}$ to take a value from the set $\{0,1, \ldots, 2 m k-1\}$ and the variables $y_{i}$ from the set $\{2 m k, 2 m k+1, \ldots, 3 m k-1,3 m k+1, \ldots, 4 m k\}$. On the other hand, according to constraints (6)-(8) each variable $e_{i}$ is defined as the difference between a variable $x$ and a variable $y$. Therefore, considering the admissible domain for $x$ and $y, e_{i}$ must have a value from the set $\{1,2, \ldots, 4 m k\}$.

According to constraint (17), a total of $4 m k$ of the $r_{i j}$ variables are equal to one and the rest of them are equal to zero. Therefore, if all of the terms in $\sum_{j=1}^{4 m k} r_{i j}$ are not equal to zero, there must be $i \in\{1,2, \ldots, 4 m k\}$ such that $\sum_{j=1}^{4 m k} r_{i j}=0$. Constraint (15) will force the corresponding $e_{i}$ to be equal to zero, which contradicts the admissible domain $e_{i}$, for $i=1,2, \ldots, 4 m k$. Therefore, the result is at hand.

Lemma2. In the propose model, constraint (13) is redundant and can be dropped.

Proof by contradiction. Assume that the constraint (13) does not hold if it gets removed.

Constraint (14) causes that only $2 m k$ variables of $w_{i j}, j=2 m k+1,2 m k+2, \ldots, 4 m k$, are equal to one and the rest of them are zero. If the constraint (13) is violated, there must be $i \in\{1,2, \ldots, 2 \mathrm{mk}\}$ such that $\sum_{j=2 m k+1}^{4 m k} w_{i j}=0$. Considering equation (12), $y_{i}$ must be equal to zero. Constraints (6)(8) define the variables $e_{i}$ as the difference between variables $x$ and $y$ and each variable $y$ appear in two of constraints (6)-(8) and since the variables $x$ take their values from the set $\{0,1, \ldots, 2 m k-1\}$ and are different from each other, $y_{i}=0$ causes at least one of the variables $e$ to be negative, which contradicts constraint (15). Therefore, the proof is complete.

The $\alpha$-labeling problem is a feasibility problem and the proposed model does not have an objective function and tries only to find a feasible solution. The proposed model has $8 \mathrm{mk}$ continuous variable and $24 m^{2} k^{2}$ binary variables and by dropping constraints (13) and (16) it has $22 m k$ constraints.

\section{Tabu Search Algorithm for the Problem}

Graph labeling problem belongs to the NP-complete class of problems. Acharya et al., proved it by using the graph coloring problem [3]. As a consequence of complex nature of this class of problems, no exact method can solve such a problem in a reasonable amount of time specially when the problem is large. During the past two decades, meta-heuristic algorithms have been used to solve many combinatorial optimization problems of this type.

Tabu search (TS) is a meta-heuristic algorithm that can be used to solve these problems [12]. This algorithm does not guarantee optimality in optimality, class of problems, but in many experiments it has been able to obtain near optimal solutions in a reasonable amount of time. However, our problem 
is in feasibility class of problems and requires no objective function and so TS requires to generate only feasible solutions.

TS starts with an initial solution and explores its neighborhood. Among these neighborhood solutions, TS chooses the best solution and moves to it. The search procedure continues until the termination criteria are satisfied. In order to avoid from getting trapped in a local optimal solution, TS uses a short term memory. This short term memory keeps track of a certain number of moves that have been performed recently. Specifically, these moves are saved in a list called "Tabu List". In each move, if the move is in the tabu list, it will not be performed and the algorithm will search for another move, except when the "Aspiration Criteria" are met. For more information and details of TS algorithms, see [11] and [12].

In the following sections, the general steps of the proposed tabu search for solving our problem are illustrated.

\subsection{Solution Procedure and General Structure of Algorithm}

The proposed tabu search algorithm starts with an initial solution with different vertex labels and tries to generate different edge labels. Two strings with the length of $2 m k$ are used to save the labels of small and large vertices. Using these two strings, another string with the length of $4 m \mathrm{k}$ is defined to save edge labels to compute the deviation value of the solution.

The general procedure of the proposed TS is as follows:

Step 1:

1-1 Choose an initial solution $x_{0}$.

1-2 Set $F^{*} \leftarrow F\left(x_{0}\right), x^{*} \leftarrow x_{0}, n=0$.

1-3 Build two tabu lists for small and large vertices.

Step 2: Set $n=n+1$. Consider $x_{n-1}$ as the current solution.

2-1 Set: $x_{n_{0}^{s}} \leftarrow x_{n-1}$ and for $n_{i}^{s}=1,2, \ldots$, NSmall , consider $x_{n_{i-1}^{s}}$ as the current solution.

2-1-1 Choose the best $\bar{x}$ that can be obtained by swapping two vertices of $x_{n_{i-1}^{s}}$ and meet one of the following conditions:

(a) the move $\bar{x} \leftarrow x_{n_{i-1}^{s}}$ is not in tabu list.

(b) $F(\bar{x})<F^{*}$.

2-1-2 Set $\bar{x} \longrightarrow x_{n_{i-1}^{s}}$.

2-1-3 If $F(\bar{x})<F^{*}$ then set $\bar{x} \rightarrow x^{*}$ and $F(\bar{x}) \rightarrow F^{*}$. If $F^{*}=0$ then go to Step 3 .

2-1-4 Update the tabu list.

2-2 Set $x_{n_{N S m a l l}^{s}} \rightarrow x_{n_{0}^{l}}$. Perform all of the operations in step 2-1 for $n_{i}^{l}=1,2, \ldots$, NLarge and perform the neighborhood search on large vertices.

Step 3: If the termination conditions are satisfied then stop, else go to Step 2.

In this study, the termination criteria is assumed to be obtaining a feasible solution or reaching a certain number of iterations. The steps of this algorithm is illustrated in more details in the following sections. 


\subsection{Initial Solution}

The initial solution is obtained according to Figure 3. In this solution, in each cycle at most one edge label is repeated and if $m$ is odd, then in the cycle with the labels on the sides of the missing value, no edge label is repeated. Edge labels are not repeated in any other cycle, and therefore, this initial solution with $m$ edge label can be a good initial solution.

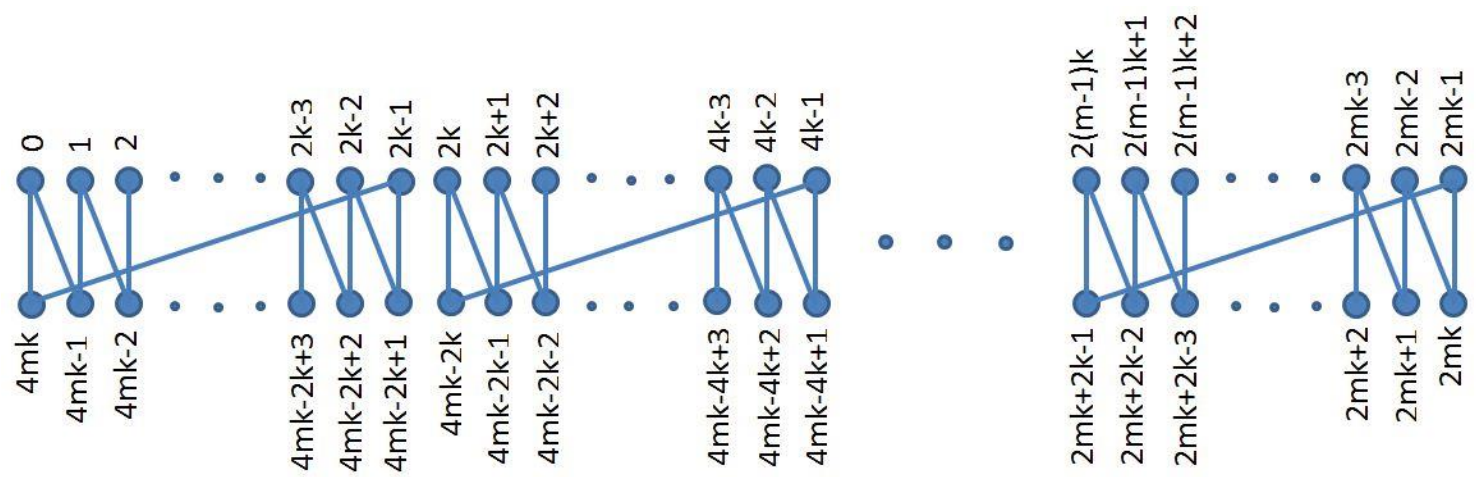

Figure 3. Initial solution

\subsection{Deviation Function}

The first idea to define a deviation function is to minimize the number of edge labels that have not been used. But this deviation function cannot be a good function for the selected solution structure. The reasons are as follows:

- A sample space of all of the edge labels between two adjacent vertices includes $n(S)=$ $2 m k * 2 m k=4 m^{2} k^{2}$ members that have the same probability.

- The edge label 1 can only be produced between vertex labels $2 m k-1$ and $2 m k$, and therefore, its probability is $p(A)=\frac{n(A)}{n(S)}=\frac{1}{4 m^{2} k^{2}}$.

- The edge label 2 can only be produced between the vertex labels $2 m k-2$ and $2 m k$, or $2 m k-1$ and $2 m k+1$, and therefore, its probability is $p(A)=\frac{n(A)}{n(S)}=\frac{2}{4 m^{2} k^{2}}$.

- As a result for other edge labels, the probabilities are presented in Table 1.

Table1. Probability of generating edge labels.

\begin{tabular}{|c|c|c|c|c|c|c|c|c|}
\hline Edges label & $1,4 m k$ & $2,4 m k-1$ & $\ldots$ & $m k, 3 m k+1$ & $m k+1,3 m k$ & $m k+2,3 m k-1$ & $\ldots$ & $2 m k, 2 m k+1$ \\
\hline $\begin{array}{c}\text { Number of possible } \\
\text { generations }\end{array}$ & 1 & 2 & $\ldots$ & $m k$ & $m k$ & $m k+1$ & $\ldots$ & $2 m k-1$ \\
\hline probability & $\frac{1}{4 m^{2} k^{2}}$ & $\frac{2}{4 m^{2} k^{2}}$ & $\ldots$ & $\frac{m k}{4 m^{2} k^{2}}$ & $\frac{m k}{4 m^{2} k^{2}}$ & $\frac{m k+1}{4 m^{2} k^{2}}$ & $\ldots$ & $\frac{2 m k-1}{4 m^{2} k^{2}}$ \\
\hline
\end{tabular}

Also,

$$
E(x)=\frac{1}{4 m^{2} k^{2}} \sum_{i=1}^{m k} i(i+4 m k+1-i)+\sum_{i=m k}^{2 m k-1} i(i+1+4 m k+i)=\frac{4 m k+1}{2} .
$$


Therefore, every pair of solutions that have the same number of absent edge labels should not have the same deviation values, because the probabilities of different edge labels are not the same and the solution with very large or small absent edge labels are unlikely to generate good solutions and cause infeasibility.

The deviation function defined in this study considers this issue and tries to assign a higher deviation value for the solutions that are less probable to be in a feasible solution. This function is as follows:

$$
s=\sum_{i=1}^{4 m k} \operatorname{Exist}(i) *|2 m k-i|+\alpha \sum_{i=1}^{4 m k} \operatorname{Exist}(i)
$$

where Exist $(i)$ is a binary variable and is equal to one if the edge label $i$ has been produced, and is equal to zero, otherwise. The value $\alpha$ determines the influence level of the previous deviation function. In this study, $\alpha$ is set to be equal to $(m * k) / 2$.

\subsection{Neighborhood Structure}

In the proposed algorithm, two neighborhood generation schemes are used. It is assumed that small (large) vertex labels are fixed and the neighborhood solutions are generated by swapping the large (small) vertex labels and the best solution that is not in the tabu list or it is but satisfies the aspiration criteria is selected as the next solution.

In each neighborhood, the number of vertices that must be swapped with each other is equal to $2 m k$. Therefore, $\quad\left(\begin{array}{c}2 m k \\ 2\end{array}\right)=\frac{(2 m k)(2 m k-1)}{2}$ neighborhood solutions should be generated in each iteration. In (20), in order to compute the deviation function, only the number of repetitions for each edge label is needed, if only two vertex labels are swapped and the others remain unchanged. Instead of computing the deviation function from scratch, the number of repetitions for each edge can be easily computed using the edge labels of the previous solution. Figure 4 illustrates this concept. After exchanging the vertices $v_{1}$ and $v_{2}$, the edge labels $\left|v_{1}-v_{1 l}\right|,\left|v_{1}-v_{1 r}\right|,\left|v_{2}-v_{2 l}\right|,\left|v_{2}-v_{2 r}\right|$ are replaced by $\left|v_{2}-v_{1 l}\right|,\left|v_{2}-v_{1 r}\right|,\left|v_{1}-v_{2 l}\right|,\left|v_{1}-v_{2 r}\right|$, respectively.

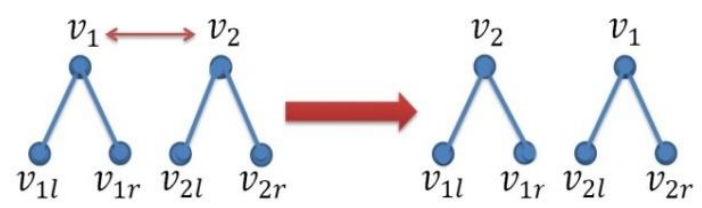

Figure 4. Swapping the two vertices' labels

\subsection{Tabu List and Tabu Tenure}

As mentioned before in Section 3.4, in one of the neighborhood generation schemes, the small vertex labels remain unchanged and swapping is performed only on the large vertex labels. Therefore, two tabu lists are used to maintain the recently performed moves, one for small vertex labels and one for large vertex labels. When two vertex labels are swapped, one of them is maintained in the tabu list for a certain number of iterations. The tabu list works in a LIFO order, meaning that when a new member is added to the list, the oldest member is removed from the list and the length of the list will 
always remain fixed. If the solution is in the tabu list but decreases the best current deviation function value, it will be removed from the list according to the "Aspiration Criteria".

\section{Special Structures for Fixing the Labels of Some Vertices}

Here, in order to improve the performance of the mathematical model and the proposed tabu search algorithm, the labels of some vertices are fixed and the other vertices are labeled such that the constraints of $\alpha$-labeling are satisfied for all the vertices. Since some labels of vertices are fixed, the labels of their edges are generated at once and no further search is needed. Therefore, the number of variables corresponding to their vertex and edge labels decrease. This cause a better efficiency of the tabu search and a decrease in the search space.

Therefore, two approaches are followed to fix vertex labels. In the first approach, two cycles are assumed to be fixed and the other vertices of the graph are labeled using the proposed algorithm. In this case, the labels of two cycles are fixed according to Figs. 5 and 6.

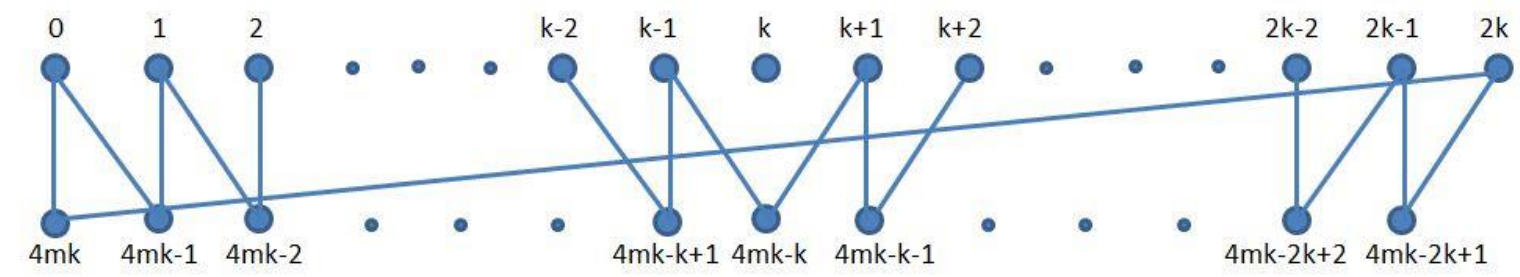

Figure 5. Fixing the labels of the first cycle

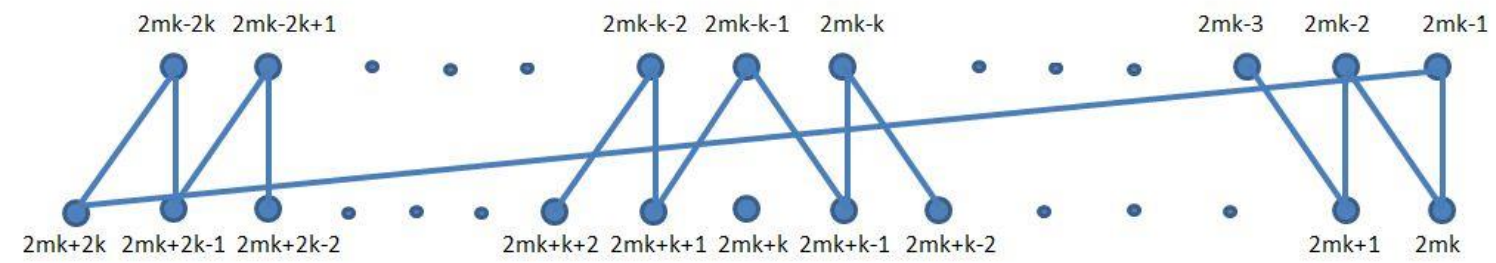

Figure 6. Fixing the labels of the second cycle

This type of fixing labels for vertices is a common approach in graph labeling and is called standard labeling $[4,6,9]$. By fixing the labels of these cycles, the edge labels $4(m-1) k+$ $1,4(m-1) k+2, \ldots, 4 m k$ in the first cycle and the edge labels $1,2, \ldots, 4 k$ in the second label are produced.

In the second policy of fixing the vertex labels, the labels of four cycles are fixed. In this case in addition to the 2 cycles presented in Figs. 4 and 5, two other cycles are also fixed according to Figs 7 and 8 . 


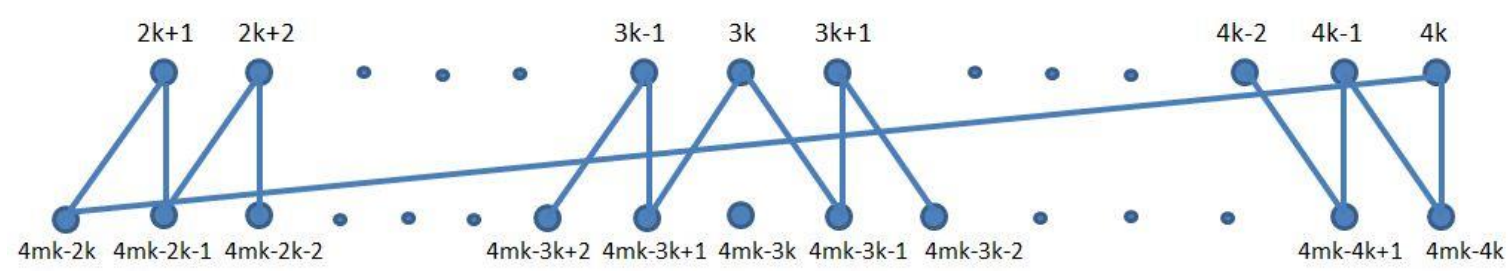

Figure 7. Fixing the labels of the third cycle

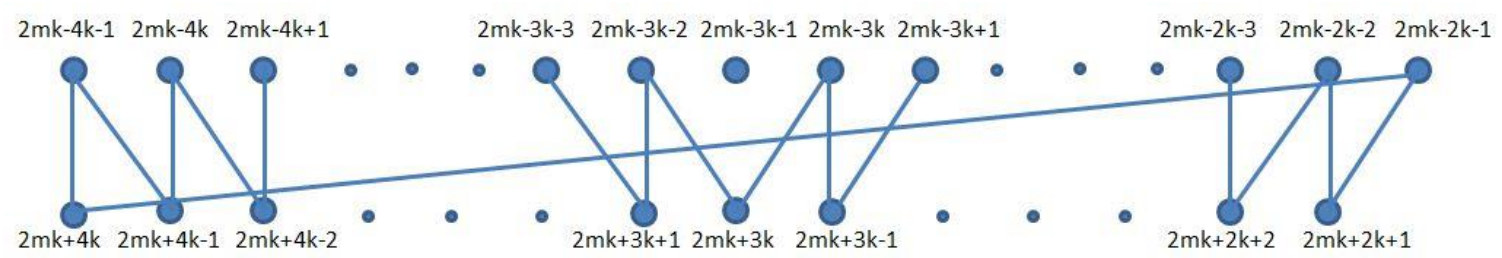

Figure 8. Fixing the labels of the fourth cycle

By fixing these cycles, the edge labels $4(m-2) k, 4(m-2) k+1, \ldots, 4(m-1) k-1$ in the third cycle and the edge labels $4 k+2,4 k+3, \ldots, 8 k+1$ in the fourth cycle are obtained. In labeling procedure for other vertices, these edge labels should not be produced.

Both approaches described in this section can generate a series of edge labels that were very time consuming if we used a search technique or a mathematical programming model. Furthermore, this can help us to extend the class of graphs having certain types of labeling [6].

\section{Computational Results}

All computations in this section are performed on a 32 bit operating system with CPU of Core 2 Due $2.10 \mathrm{GHz}$ and $3.00 \mathrm{~GB}$ of RAM. The integer programming model proposed in Section 2 has been modeled using IBM ILOG CPLEX 12.2 software and the running time for each graph is reported in Table 2.

By running the integer programming model and removing the constraints (13) and (16), it is observed that the running time of the algorithm increases. In fact, these redundant constraints can act as cutting planes in the solution space and delete some infeasible solutions, and turn to cause a better running time. These constraints, without omission of any feasible integer point, shrink the search space of feasibility type problem like ours and help the branch and cut algorithm employed by the software to reach the final feasible solution faster. Results of the model after adding these constraints are presented in Table 2.

The proposed tabu search algorithm was coded in C++ using Visual Studio 2014 compiler and the required time for each graph is presented in Table 3. The NSmall and NLarge in the proposed tabu search algorithm are assumed to be equal to each other and are performed with values of 1, 3 and 5. Obviously, if the algorithm reaches the feasible solution for 1 or 3 , it will not be run with a larger value. The size of the tabu list for each value of NSmall or NLarge varies from 1 to 5 until the algorithm reaches a feasible solution. Termination criteria for each value of NSmall and NLarge and tabu tenure is considered to be performing 10000 iterations for graph with 250 or less vertices and 100000 iterations for other graphs or reaching a feasible solution. 
In these tables, the symbols ' $\alpha$ ' and ' $\beta$ ' denote that two and four cycles are fixed respectively. The integer programming model for $3 \mathrm{C} 4$, or $Q(1,3)$, shows that there is no feasible labeling for this graph in less than 1 second. Also, the tabu search algorithm shows that no feasible solution for this problem exists in a reasonable amount of time. This result is the same as we have known from the literature [4]. In Table 2, if the CPLEX cannot reach a feasible solution for a graph in 900 seconds, a '-' character is reported.

Table 2. Time of $\boldsymbol{\alpha}$-labeling of $\boldsymbol{Q}(\boldsymbol{m}, \mathbf{4} \boldsymbol{k})$ by CPLEX (in

\begin{tabular}{|c|cccc|}
\hline$m k$ & 1 & 2 & 3 & 4 \\
\hline 1 & 1 & 1 & 1 & 1 \\
2 & 1 & 1 & 11 & 238 \\
3 & $N$ & 62 & - & - \\
4 & 1 & $25^{\alpha}$ & $290^{\alpha}$ & - \\
5 & 86 & $97^{\alpha}$ & $834^{\alpha}$ & - \\
6 & 169 & $769^{\alpha}$ & $43^{\beta}$ & $123^{\beta}$ \\
7 & 474 & $548^{\beta}$ & $734^{\beta}$ & - \\
8 & - & $253^{\beta}$ & - & - \\
\hline
\end{tabular}

Table 3. Time of $\boldsymbol{\alpha}$-labeling of $\boldsymbol{Q}(\boldsymbol{m}, \mathbf{4 k})$ by tabu search (in seconds).

\begin{tabular}{|c|cccccccccc|}
\hline$m k$ & 1 & 2 & 3 & 4 & 5 & 6 & 7 & 8 & 9 & 10 \\
\hline 1 & 1 & 1 & 1 & 1 & 1 & 1 & 1 & 1 & 1 & 1 \\
2 & 1 & 1 & 1 & 1 & 1 & 1 & 6 & 8 & 10 & 112 \\
3 & $N$ & 1 & 1 & 2 & 14 & 12 & 21 & 23 & 38 & 49 \\
4 & 1 & 1 & 2 & 17 & 201 & 20 & 50 & 174 & 35 & 6 \\
5 & 1 & 1 & 3 & 27 & 30 & 45 & 44 & 56 & 239 & 117 \\
6 & 1 & 1 & $3^{\beta}$ & $1^{\beta}$ & $1^{\beta}$ & $1^{\beta}$ & $1^{\beta}$ & $3^{\beta}$ & $4^{\beta}$ & $1^{\beta}$ \\
7 & 28 & $1^{\beta}$ & $1^{\beta}$ & $1^{\beta}$ & $1^{\beta}$ & $1^{\beta}$ & $1^{\beta}$ & $1^{\beta}$ & $1^{\beta}$ & $4^{\beta}$ \\
8 & 65 & $1^{\beta}$ & $1^{\beta}$ & $1^{\beta}$ & $1^{\beta}$ & $3^{\beta}$ & $1^{\beta}$ & $7^{\beta}$ & $4^{\beta}$ & $72^{\beta}$ \\
9 & 10 & $1^{\beta}$ & $1^{\beta}$ & $1^{\beta}$ & $1^{\beta}$ & $1^{\beta}$ & $72^{\beta}$ & $52^{\beta}$ & $953^{\beta}$ & $1170^{\beta}$ \\
10 & 182 & $1^{\beta}$ & $1^{\beta}$ & $7^{\beta}$ & $10^{\beta}$ & $14^{\beta}$ & $660^{\beta}$ & $57^{\beta}$ & $1123^{\beta}$ & $3659^{\beta}$ \\
& & & & & & & & & & \\
\hline
\end{tabular}

The tabu search algorithm in each iteration moves to the best neighborhood solution if it is not in tabu list. This new solution may have a worse deviation value than the previous one, and therefore, the values of the deviation function are not necessarily decreasing throughout the run and they fluctuate around a certain point until zero is reached, a feasible solution, or the maximum iteration numbers. Fig. 9 presents the values of the deviation function for different iterations for labeling $Q(9,40)$ with $N$ Small $=$ Nlarge $=1$ and the tabu list length of 2 . The algorithm reaches the feasible solution after 8611 iterations. Furthermore, Fig. 10 shows these values for $Q(10,40)$ and NSmall $=$ Nlarge $=1$ and the tabu list length of 3 . As seen in these figures, the proposed algorithm reaches the feasible labeling without any discernible trend in consecutive iterations.

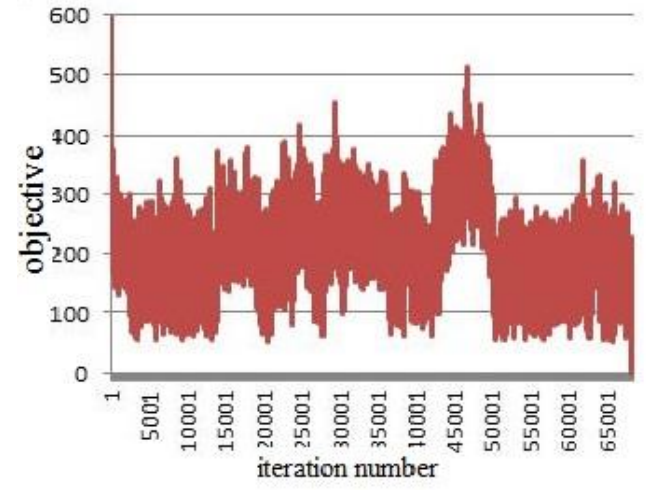

Figure 9. Deviation value of graph $\boldsymbol{Q}(\mathbf{1 0}, \mathbf{4 0})$ by $\boldsymbol{N S m a l l}=$ Nlarge $=\mathbf{1}$ and the tabu list length being 3

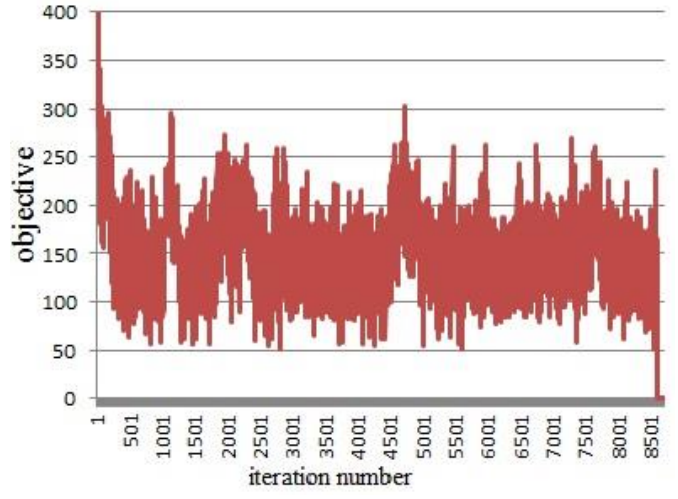

Figure 10. Deviation value of graph $Q(9,40)$ by $\boldsymbol{N S m a l l}=\mathbf{N l a r g e}=\mathbf{1}$ and the tabu list length being 2 
Theorem. The quadratic graph $Q(m, 4 k), 1 \leq m, k \leq 10$, has an $\alpha$-labeling with the exception of $m=3, k=1$.

Proof. The result is proved by obtaining the labeling for all possible cases not reported in the literature. The $\alpha$-labeling for $Q(m, 4 k)$ with $6 \leq m \leq 10,2 \leq k \leq 10$ are presented, in the appendix. For the remaining cases, see [5], [13] and [14], to complte the proof.

\section{Conclusion}

Here, first, an integer programming model was proposed to find $\alpha$-labeling of quadratic graphs $Q(m, 4 k)$. By solving the integer programming model, $\alpha$-labelings for graphs with at most 96 vertices were obtained. Then, a tabu search algorithm was proposed. The computational results showed that the proposed tabu search algorithm performed better than the integer programming model and $\alpha$-labeling for $Q(m, 4 k)$ graphs with $m, k \leq 10$, with the exception of $m=3, k=1$, were generated in pretty short times. The major contributions of our work can be summarized in three parts: first, modeling and solving a difficult graph theory problem by a mathematical programming model. Next, an example of a feasibility class of problems like graph labeling in which we want to find a feasible solution, rather than optimal solution, was given. Finally, it was shown that how a metaheuristic algorithm could solve the problem. As known, in most optimality class of problems, metaheuristics can generate near optimal solutions but there is no guarantee that they can reach an optimal solution. However, here, using a metaheuristic algorithm, like tabu search, we were able to generate $\alpha$-labelings of all the desired cases.

\section{Appendix}

In this section labeling for graph $m C_{4 k}$ with $6 \leq m \leq 10,2 \leq k \leq 10$ is presented. The labeling for $m=6, k=2$ is as follows:

$m=6, k=2:(2-42-5-44-6-41-7-38)-(8-35-9-39-10-43-11-32)-(12-29-13-33-14-37-15-40)-(16-$ 26-17-31-18-30-19-34) - (0-48-1-47-3-46-4-45) - (20-28-21-27-22-25-23-24)

For the other graphs, labels of the following four cycles are assumed to be fixed:

First cycle:

$0,4 m k, 1,4 m k-1, \ldots, k-1,(4 m-1) k+1, k+1,(4 m-1) k, k+2,(4 m-1) k-1, \ldots, 2 k,(4 m-$ $2) k+1$.

Second cycle

$2 k+1,(4 m-2) k, 2 k+2,(4 m-2) k-1, \ldots, 3 k,(4 m-3) k+1,3 k+1,(4 m-3) k-1,3 k+2,(4 m-$ $3) k-2, \ldots, 4 k,(4 m-4) k$.

Third cycle:

$(2 m-4) k-1,(2 m+4) k,(2 m-4) k,(2 m+4) k-1, \ldots,(2 m-3) k-2,(2 m+3) k+1$,

$(2 m-3) k,(2 m+3) k,(2 m-3) k+1,(2 m+3) k-1, \ldots,(2 m-2) k-1,(2 m+2) k+1$. 
Fourth cycle:

$(2 m-2) k,(2 m+2) k,(2 m-2) k+1,(2 m+2) k-1, \ldots,(2 m-1) k-1,(2 m+1)+1,(2 m-$ 1) $k,(2 m+1) k-1,(2 m-1) k+1,(2 m+1) k-2, \ldots, 2 m k-1,2 m k$.

For the remaining $m-4$ cycles, the labels are presented in Table 4 .

Table 4. $\alpha$-labelings of the remaining cycles of $Q(m, 4 k)$.

\begin{tabular}{|c|c|c|}
\hline$m$ & $\boldsymbol{k}$ & $\alpha$-labelings of remaining $\boldsymbol{m}-\mathbf{4}$ cycles \\
\hline 6 & 3 & $(63-3-50-20-59-14-58-21-49-22-53-17),(55-15-57-16-51-19-52-18-56-13-39-26)$ \\
\hline 6 & 4 & $\begin{array}{l}(84-4-67-27-79-17-76-19-77-21-75-20-69-22-73-23),(74-26-71-28-70-24-68-30-65-29-66-25-78-18-52 \\
-35)\end{array}$ \\
\hline 6 & 5 & 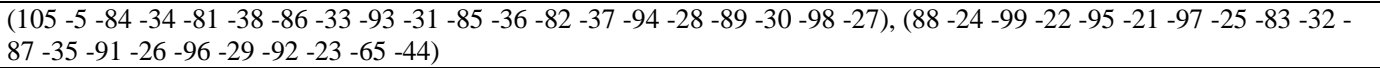 \\
\hline 6 & 6 & 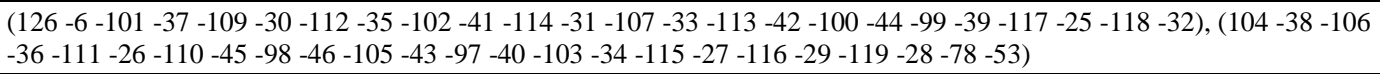 \\
\hline 6 & 7 & $\begin{array}{l}(147-7-118-52-117-54-113-51-115-42-134-34-139-30-131-35-133-40-129-44-130-48-116-49-119-32-136 \\
-37),(122-43-123-47-124-41-125-53-114-45-135-38-132-29-137-31-138-36-127-39-120-46-121-50-128- \\
33-91-62)\end{array}$ \\
\hline 6 & 8 & 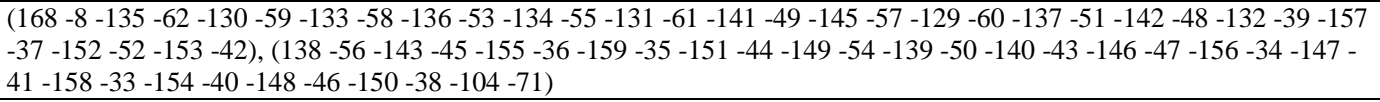 \\
\hline 6 & 9 & 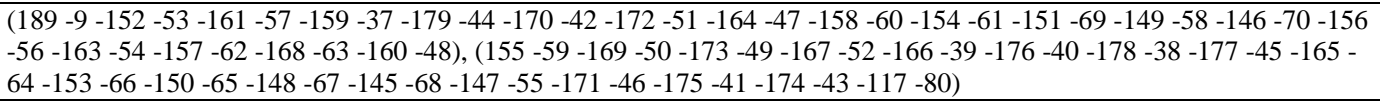 \\
\hline 6 & 10 & 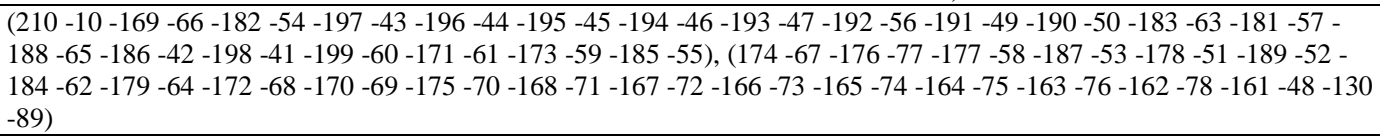 \\
\hline 7 & 2 & $(50-2-41-15-46-9-47-14),(43-13-38-18-37-16-40-11),(44-10-45-17-39-12-30-21)$ \\
\hline 7 & 3 & $\begin{array}{l}(75-3-62-13-70-14-67-22-69-15-61-17),(65-23-64-21-60-20-71-16-68-18-66-28),(59-24-58-25-57-26- \\
56-27-55-19-45-32)\end{array}$ \\
\hline 7 & 4 & $\begin{array}{l}(100-4-83-27-93-19-94-18-91-20-92-24-82-21-90-23),(86-22-87-38-73-25-95-17-80-28-81-31-85-30- \\
89-29),(79-32-78-33-77-34-76-35-75-36-74-37-88-26-60-43)\end{array}$ \\
\hline 7 & 5 & 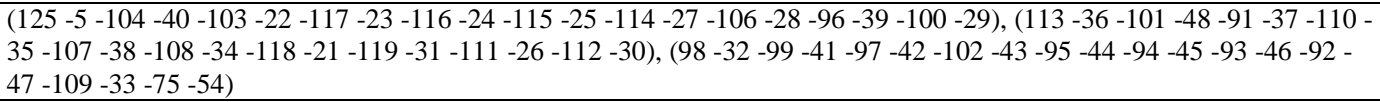 \\
\hline 7 & 6 & 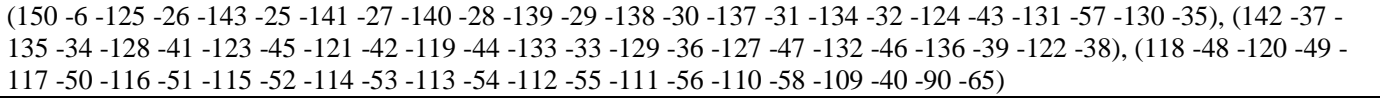 \\
\hline 7 & 7 & 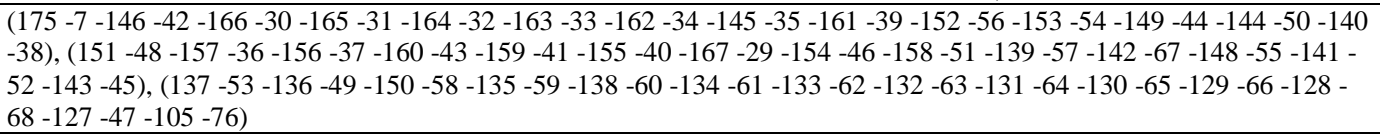 \\
\hline 7 & 8 & 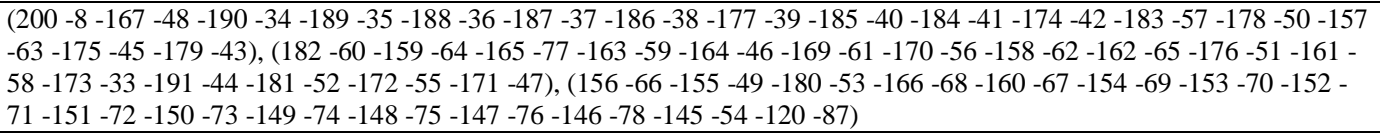 \\
\hline 7 & 9 & 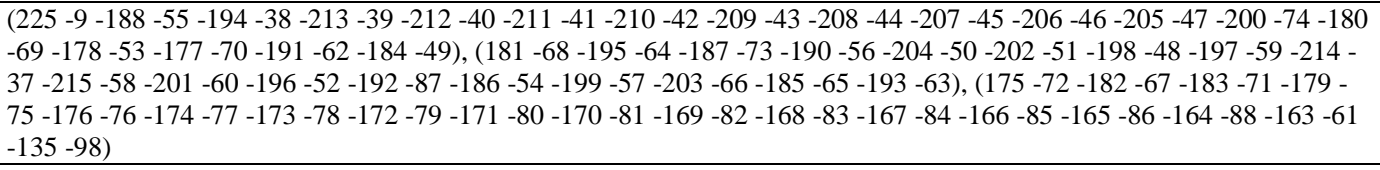 \\
\hline 7 & 10 & 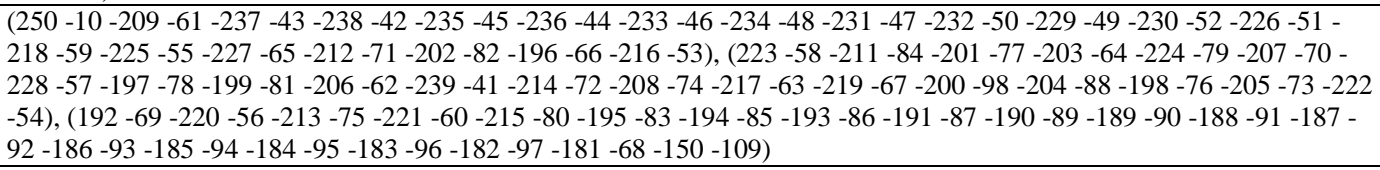 \\
\hline 8 & 2 & $\begin{array}{l}(58-2-49-17-46-19-43-13),(54-12-52-11-45-14-53-10),(55-9-47-22-50-15-51-18),(44-21-41-20-42-16- \\
34-25)\end{array}$ \\
\hline 8 & 3 & $\begin{array}{l}(87-3-74-13-82-14-81-15-80-16-79-17),(76-24-69-26-67-27-75-19-77-18-78-21),(70-23-73-20-71-34- \\
83-29-68-22-66-28),(65-30-64-31-63-32-62-33-61-25-51-38)\end{array}$ \\
\hline
\end{tabular}


Table 4. Continued

\begin{tabular}{|c|c|c|}
\hline$m$ & $\boldsymbol{k}$ & $\alpha$-labelings of remaining $m-4$ cycles \\
\hline 8 & 4 & 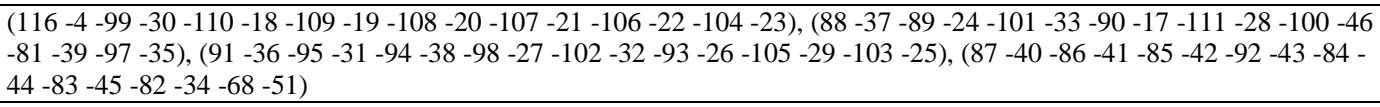 \\
\hline 8 & 5 & 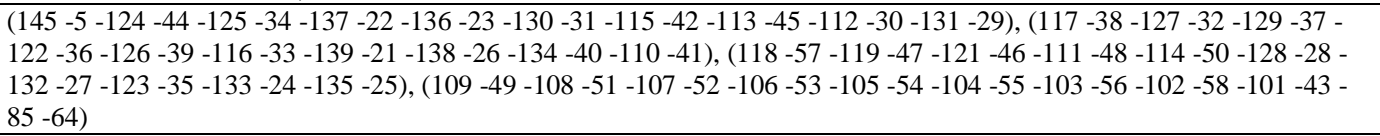 \\
\hline 8 & 6 & 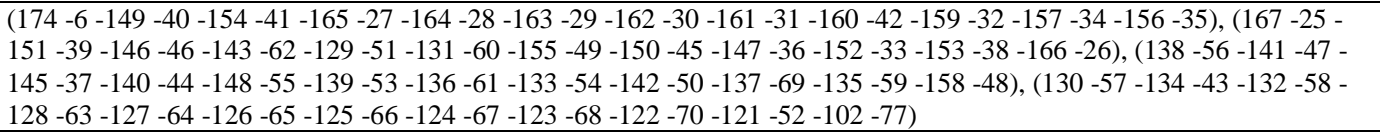 \\
\hline 8 & 7 & $\begin{array}{l}(203-7-174-47-194-30-193-31-192-32-191-33-190-34-189-35-188-36-187-37-186-54-185-39-183-40-179 \\
-38),(159-80-164-46-184-42-178-48-173-45-180-59-169-43-177-29-195-50-172-52-171-63-167-53-176- \\
60-166-57),(160-66-158-58-163-64-157-56-151-55-170-41-181-44-156-49-182-65-162-51-175-62-165- \\
67-155-68-154-69),(153-70-152-71-161-72-150-73-149-74-148-75-147-76-146-77-145-78-144-79-143- \\
81-142-82-141-61-119-90)\end{array}$ \\
\hline 8 & 8 & 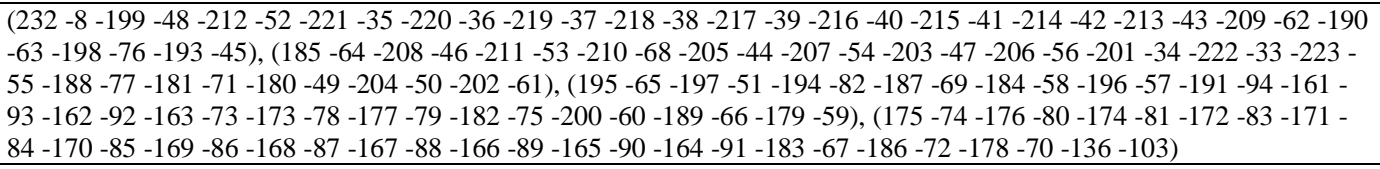 \\
\hline 8 & 9 & 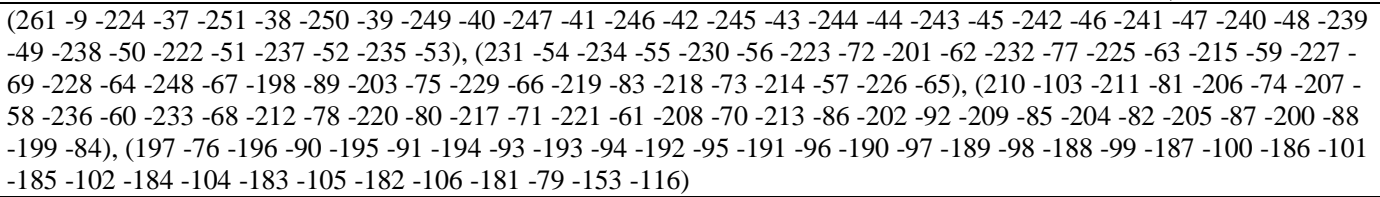 \\
\hline 8 & 10 & 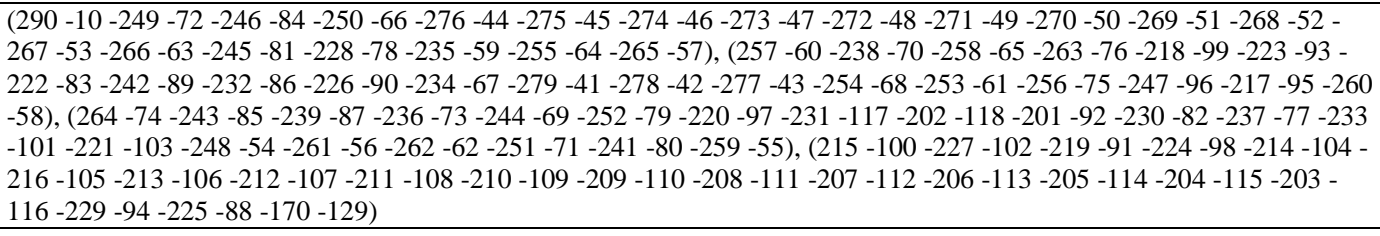 \\
\hline 9 & 2 & $\begin{array}{l}(66-2-57-24-58-18-50-12),(45-25-48-19-46-22-47-26),(56-15-52-21-49-23-53-17),(60-13-61-10-55-11- \\
63-14),(51-16-59-9-62-20-38-29)\end{array}$ \\
\hline 9 & 3 & $\begin{array}{l}(99-3-86-13-94-14-93-15-92-16-84-17),(90-24-89-19-79-22-91-20-95-21-77-18),(82-28-78-29-80-40- \\
83-25-88-33-75-30),(76-32-85-23-87-26-74-27-73-34-72-35),(71-36-70-37-69-38-68-39-67-31-57-44)\end{array}$ \\
\hline 9 & 4 & $\begin{array}{l}(132-4-115-30-126-18-125-19-124-20-123-21-122-22-120-23),(105-31-118-34-114-45-100-36-117-24- \\
116-17-127-37-97-44),(106-38-109-32-121-26-98-33-111-29-112-39-96-46-102-43),(113-27-103-54-89- \\
41-99-47-101-35-110-40-107-28-119-25),(95-48-94-49-93-50-92-51-91-52-90-53-104-42-76-59)\end{array}$ \\
\hline 9 & 5 & 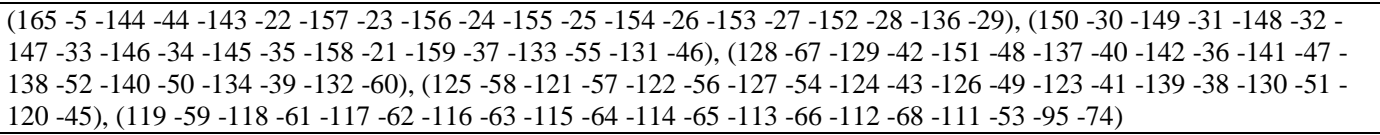 \\
\hline 9 & 6 & 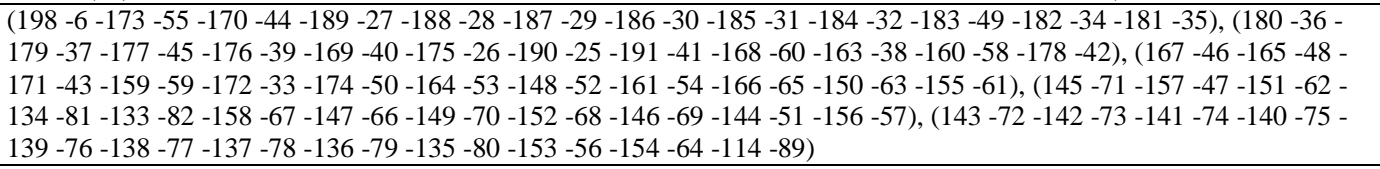 \\
\hline 9 & 7 & 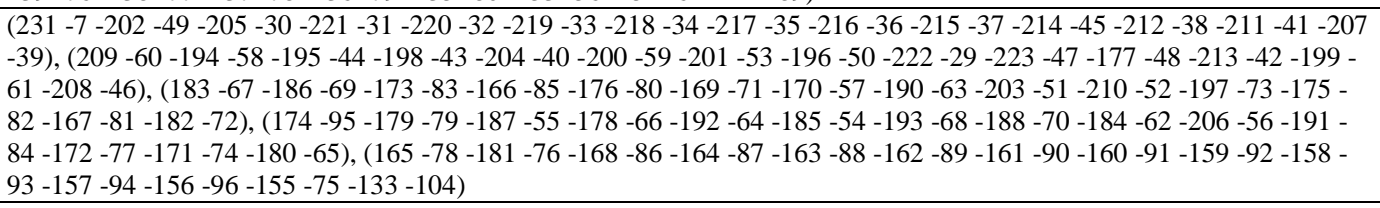 \\
\hline 9 & 8 & 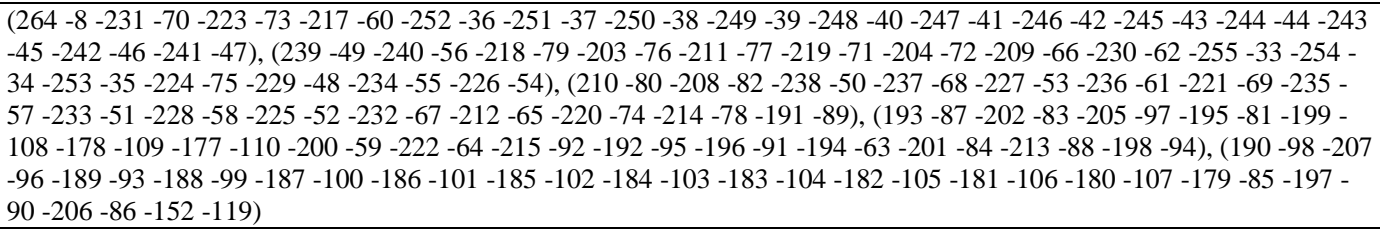 \\
\hline
\end{tabular}


Table 4. Continued

\begin{tabular}{|c|c|c|}
\hline$m$ & $\boldsymbol{k}$ & $\alpha$-labelings of remaining $m-4$ cycles \\
\hline 9 & 9 & 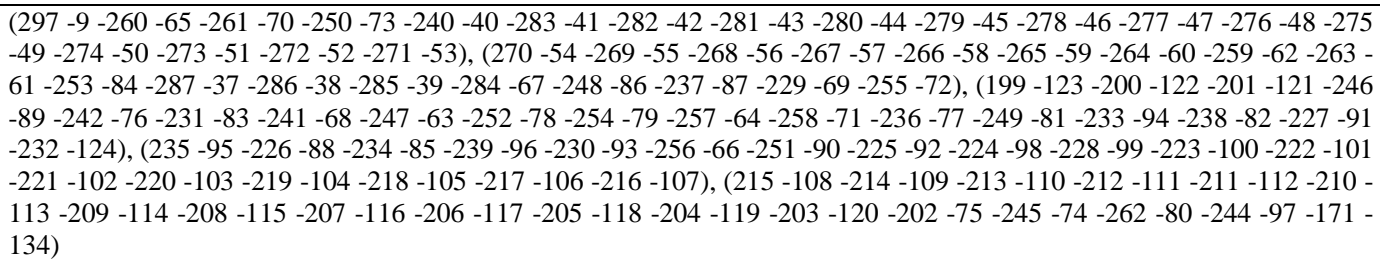 \\
\hline 9 & 10 & $\begin{array}{l}330-10-289-73-285-71-291-89-279-74-315-45-314-46-313-47-312-48-311-49-310-50-309-51-308-52-307 \\
-53-306-54-305-55-304-56-303-57-302-58-301-59),(299-60-300-62-298-61-296-64-297-63-294-66-295- \\
65-292-68-286-67-293-78-272-94-319-41-318-42-317-43-316-44-266-84-264-100-271-88-276-119-263- \\
76),(273-70-287-77-277-85-274-97-267-107-261-75-282-69-290-79-283-86-260-81-280-95-256-98-251- \\
105-252-102-278-72-281-83-284-91-275-80-288-92-255-82),(265-99-258-96-268-87-262-106-254-103- \\
224-135-223-136-222-137-221-138-250-109-249-110-248-111-247-112-246-113-245-114-244-115-243-116 \\
-242-117-241-118-240-120),(239-121-238-122-237-123-236-93-235-124-234-125-233-126-232-127-231- \\
128-230-129-229-130-228-131-227-132-226-133-25 \\
125-134-253-101-269-104-259-90-257-108-190-149)\end{array}$ \\
\hline 10 & 2 & $\begin{array}{l}(74-2-65-17-63-12-69-15),(71-13-68-16-66-10-70-9),(55-20-64-11-54-14-61-19),(59-21-62-23-51-18- \\
67-22),(56-25-57-30-49-26-52-27),(53-29-50-28-58-24-42-33)\end{array}$ \\
\hline 10 & 3 & $\begin{array}{l}(111-3-98-13-106-14-105-15-104-16-103-17),(88-23-101-19-100-20-95-22-99-27-86-34),(93-30-83-25- \\
94-46-91-21-85-24-107-31),(89-33-84-35-92-26-97-18-102-28-96-29),(87-32-82-36-80-39-81-38-78-41 \\
-79-40),(77-42-76-43-75-44-74-45-73-37-63-50)\end{array}$ \\
\hline 10 & 4 & $\begin{array}{l}(148-4-131-34-142-18-141-19-140-20-139-21-138-22-135-23),(137-27-136-25-128-26-133-28-132-17-143 \\
-29-130-30-129-31),(122-37-121-42-118-40-114-44-117-48-125-32-124-38-134-39),(112-52-105-46-127- \\
33-108-47-115-35-126-36-123-41-113-24),(103-49-116-45-110-62-107-43-109-51-106-54-111-55-104- \\
53),(102-56-119-57-101-58-100-59-99-60-98-61-97-50-84-67)\end{array}$ \\
\hline 10 & 5 & 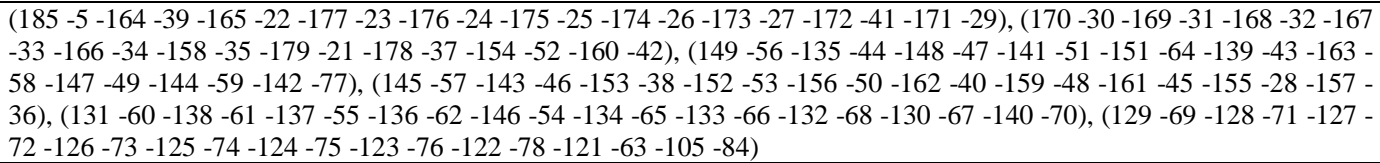 \\
\hline 10 & 6 & 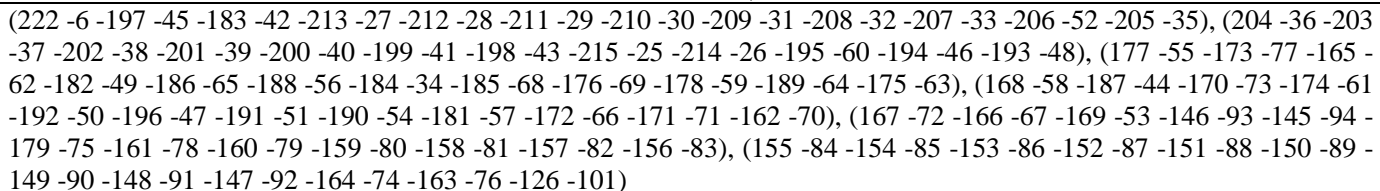 \\
\hline 10 & 7 & $\begin{array}{l}(259-7-230-62-217-55-250-31-248-32-247-33-246-34-245-35-244-36-243-37-242-38-241-39-240-40-238 \\
-41),(206-58-229-52-233-45-231-44-235-51-197-77-218-66-226-50-249-29-251-30-223-59-208-86-215- \\
64-199-81),(195-76-188-84-200-90-189-82-191-108-194-83-198-96-204-78-192-79-211-71-213-85-202- \\
75-220-70-209-72),(228-48-214-61-205-74-207-69-203-73-216-47-236-46-224-49-222-68-225-67-234-42 \\
-227-57-239-43-237-54),(201-65-212-53-232-60-221-56-219-63-187-87-182-97-193-92-190-93-196-91- \\
185-94-184-95-183-99-186-80),(181-88-180-98-179-100-178-101-177-102-176-103-175-104-174-105-173 \\
-106-172-107-171-109-170-110-169-89-147-118)\end{array}$ \\
\hline 10 & 8 & 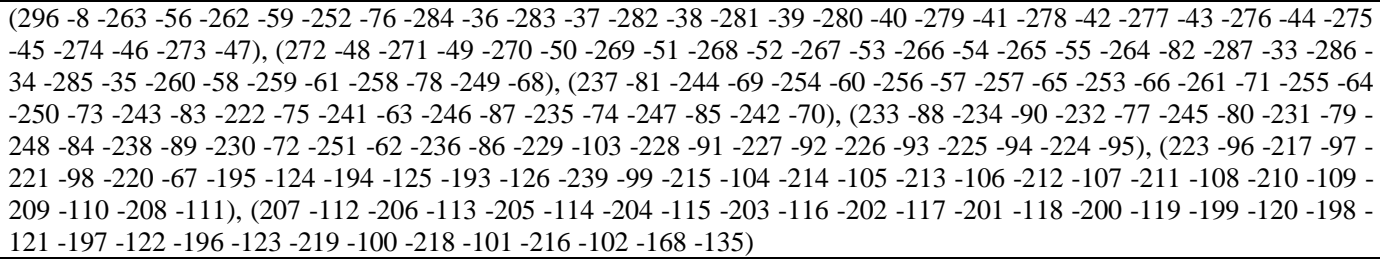 \\
\hline 10 & 9 & 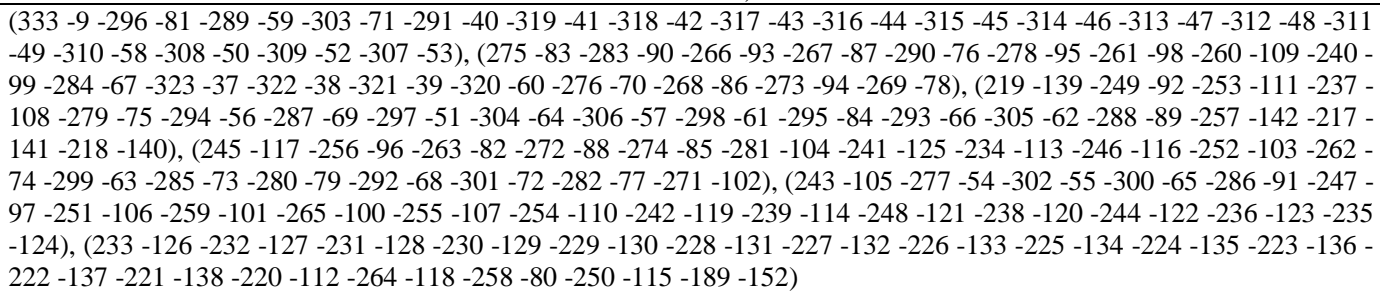 \\
\hline
\end{tabular}


Table 4. Continued

\begin{tabular}{|c|c|c|}
\hline$m$ & $\boldsymbol{k}$ & $\alpha$-labelings of remaining $\boldsymbol{m}-\mathbf{4}$ cycles \\
\hline 10 & 10 & 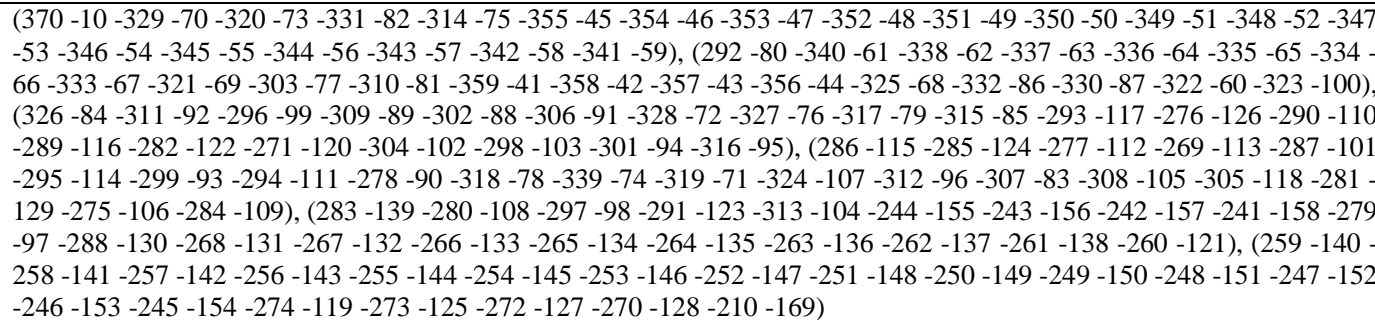 \\
\hline
\end{tabular}

\section{References}

[1] Abrham J. (1994), All 2-regular graphs consisting of 4-cycles are graceful, Discrete Mathematics, 135, 1-14.

[2] Abrham J. (1991), Extension of graceful valuations of 2-regular graphs, Cong. Numer., 84, 51-59.

[3] Abrham J., and Kotzig A. (1996), Graceful valuations of 2-regular graphs with two components, Discrete Mathematics, 150, 3-15.

[4] Acharya, B. D., Germina, K.A., Princy, K.L., and Rao, S.B. (2008), Graph labellings, embedding and NP-completeness theorems, J. Combin. Math. Combin. Comput., 67, 163180.

[5] Eshghi K. (2002), $\alpha$-valuations of special classes of quadratic graphs, Bulletin of Iranian Mathematical Society, 28(1), 29-42.

[6] Eshghi K. (1997), Existence and construction of $\alpha$-labeling of 2-regular graphs with three components, Ph.D. Thesis, University of Toronto, Ontario, Canada.

[7] Eshghi K., and Azimi P. (2007), An algorithm for finding a feasible solution of graph labeling Problems, Utilitas Math., 72, 163-174.

[8] Eshghi K., and Azimi P. (2004), Application of mathematical programming in graceful labelling problem of graphs, Journal of Applied Mathematics, 11, 1-8.

[9] Eshghi K. (2002), Introduction to Graceful Graphs, Sharif University of Technology. http://sharif.edu/ eshghi/GracefulGraphs- FinalEdition-89-12-15.pdf

[10] Gallian J. A. (2015), A Dynamic survey of graph labeling, Electronic Journal of Combinatorics, 18, 16th edition.

[11] Gendreau M., and Potvin J. Y. (2010), Handbook of Metaheuristics: Second edition, Springer.

[12] Glover F. (1990), Tabu Search: A Tutorial, Interfaces, 20(4), 74-94.

[13] Kotzig A. (1975), $\beta$-valuations of quadratic graphs with isomorphic components, Utilitas Math., 7, 263-279.

[14] Lakshmi D.R., and Vangipuram S. (1987), An $\alpha$-valuation of quadratic graph Q(4, 4k), Proc. Nat. Acad. Sco., India Sect. A57 (No. 4, Suppl.), 576-580.

[15] Mahmoudzadeh H., Eshghi K. (2010), A Metaheuristic Approach to the Graceful Labeling Problem of Graphs, International Journal of Applied Metaheuristic Computing, 4, 42-56.

[16] Redl T.A. (2003), Graceful graphs and graceful labelings: Two mathematical programming formulations and some other new results, Technical Report TR03-01, Department of Computational and Applied Mathematics, Rice University, Houston, Texas.

[17] Rosa A. (1966), On certain valuations of the vertices of a graph. In: Proceedings of the International Symposium in theory of graphs, 349-355. 
[18] Smith B.M. (2006), Constraint programming models for graceful graphs, Principles and practice of constraint programming - CP, LNCS ,Vol. 4204, 545-559.

[19] Smith B. M., Puget J.F. (2010), Constraint models for graceful graphs, Constraints, 15, 6492. 